\title{
Synthesis of carbon nano-spheres by thermal plasma treatment of polypropylene
}

Sina Mohsenian

University of Massachusetts Lowell, USA

In the last century, nano-structured carbon materials have attracted considerable attentions due to their multiple beneficial applications. These carbon nano structures have been synthesized by different technologies including laser ablation, chemical vapor deposition (CVD), plasma enhanced chemical vapor deposition (PECVD), etc. Due to operation in atmospheric pressure and being used for conversion the abundant low value materials such as solid municipal wastes to very useful materials, thermal plasma is one of the most appropriate methods for carbon nano-structured synthesis. In this study, it has been experimentally demonstrated the feasibility of producing carbon nano-spheres (CNS) from common plastic waste material (polypropylene), using thermal plasma treatment. Samples were treated in a furnace fitted with a twin dc thermal plasma torch. The resultant solid products were analyzed to determine their composition and morphology by EDS and SEM analysis. The
EDS pattern for the samples synthesized at $80 \mathrm{~A}$ illustrates that there are some kinds of impurities in solid products such as oxygen and aluminum which came from vaporization of torch's alumina ceramic nozzle. However, in the pattern obtained at $100 \mathrm{~A}$, there was just one major carbon peak. The elevated temperature plasma jet at $100 \mathrm{~A}$ arc current can vaporize all the contamination from sample and processing region, and separate them from conclusive solid products. The SEM image for $100 \mathrm{~A}$ treatment reveals that the solid carbon particles are very fine carbon nano-spheres with about $50 \mathrm{~nm}$ diameter. This method for synthesis of carbon nano-spheres may find a way to be considered in industrial waste disposal installation, since these advanced products can be obtained from very non-useful and environmentally hazardous materials which may enhance the economic efficiency of plasma waste disposal industry, significantly.

e: sinamohsenian@yahoo.com 